\title{
Stratégies conversationnelles dans le discours médical médiatisé
}

\section{Conversational strategies in mediated medical discourse}

\author{
HaYaT DJaOUdi \\ Université de Tizi-Ouzou (Argelia) \\ djaoudihayat@yahoo.fr
}

\begin{abstract}
Through this article, which falls within the disciplinary field of discourse analysis, we will try to identify the main conversational strategies deployed in mediated medical discourse, by relying on a corpus made up of an exclusive interview broadcast on the BFMTV television channel. More precisely, we will highlight the specific discursive features specific to this type of communication and will closely analyze the interactive dynamic and the specific language behaviors of the interlocutors.
\end{abstract}

\section{Keywords}

Medical discourse, media interaction, strategy, conversational analysis.

\section{Resumen}

A través de este artículo, que se enmarca en el campo disciplinar del análisis del discurso, intentaremos identificar las principales estrategias conversacionales desplegadas en el discurso médico mediado, apoyándonos en una entrevista exclusiva emitida en el canal de televisión BFMTV. Más precisamente, destacaremos las especificidades discursivas propias de este tipo de comunicación y analizaremos de cerca la dinámica interactiva y los comportamientos lingüísticos específicos de los interlocutores.

\section{Palabras clave}

Discurso médico, interacción mediática, estrategia, análisis conversacional. 


\section{Présentation du cadre théorique et méthodologique}

Notre recherche s'inscrit dans le cadre de l'analyse du discours scientifique. Plus exactement, nous nous intéressons au discours médical médiatisé par le biais de la télévision. "Le discours scientifique est entendu ici au sens de discours produit dans le cadre de l'activité de recherche à des fins de construction et de diffusion du savoir" (Fanny, 2010).

A vrai dire, nombre de spécificités et marques parsèment ce type de discours qui met en interaction un journaliste et un médecin ayant des statuts sociaux différents (éventuellement des invités sur le plateau). Une situation de rencontre et de débat ayant une finalité scientifique.

Dans le cadre de la recherche entreprise, nous nous sommes appuyés sur un corpus constitué d'un entretien exclusif diffusé sur la chaîne BFMTV discutant de la thématique relative à la Covid-19. Il convient de souligner que notre corpus est constitué d'un entretien, d'une heure de temps, réalisé par une journaliste auprès d'un spécialiste français des maladies infectieuses, professeur en microbiologie. Cette situation médiatisée, diffusée en différée, a eu lieu dans le bureau du chercheur en date du 30 avril 2020. (L'intégralité de l'entretien est disponible sur le lien <https:/www.youtube.com/watch? $\mathrm{v}=$ cj4bGVszZP8>).

Nous avons choisi de nous focaliser sur cette thématique d'actualité qui a pris de l'ampleur et a cristallisé de nombreux débats télévisés. En effet, cette crise sanitaire d'envergure a secoué le monde et a occupé une place prépondérante dans l'univers médiatique. En ce sens, ce phénomène inédit a généré une couverture médiatique exceptionnelle et sans précédent. Plus précisément, on compte déjà plusieurs mois de médiatisation du coronavirus dans les chaînes d'information. Ces dernières consacrent plus de la moitié de leurs temps d'antenne à la Covid-19.

Dans ce contexte, nous avons entrepris une recherche dont la problématique est articulée autour de ces interrogations: quelles sont les spécificités prosodiques, lexicales et stylistiques inhérentes au discours médical médiatisé? Et quelles sont les stratégies conversationnelles mobilisées par les interlocuteurs dans ce genre discursif spécifique?

Force est de préciser que le discours médical médiatisé est un discours sur la Science; il s'agit plus exactement d'expliquer des phénomènes, relater des faits ou livrer des informations scientifiques crédibles. A travers ce discours, on cherche la diffusion d'informations scientifiques auprès d'un large public.

Par ailleurs, il convient de préciser que "toute interaction médiatique se distingue de la conversation ordinaire de par son degré d'authenticité et d'interactivité” (Bennaissa, 2018: 02). Ce genre d'interaction provoque un débat d'ordre scientifique et se caractérise, à la fois, par une certaine programmation et une relative spontanéité des échanges.

En bref, l'interaction médiatique est une communication publique, elle est par excellence un espace ouvert d'échanges et de discussion entre les acteurs sociaux qui "exercent 
les uns sur les autres un réseau d'influences mutuelles. Parler c'est échanger et c'est changer en échangeant" (Kerbrat-Orecchioni, 2016: 17). Dans ce type de communication, les interlocuteurs mettent en œuvre des stratégies interactionnelles spécifiques qui "se décident sur le terrain en fonction de la dynamique particulière de l'échange" (Vion, 1992: 196).

\section{Analyse du corpus}

Nous analysons notre corpus à l'aide d'outils de l'analyse conversationnelle; cette dernière a pour objet d'étude les stratégies et les règles qui régissent le fonctionnement de la conversation. Autrement dit, l'analyse conversationnelle "étudie le fonctionnement des tours de parole, les séquences d'action, l'organisation thématique, à partir de la transcription des conversations attestées" (Siouffi \& Raemdonck, 2007: 68).

Afin de faciliter la lecture du document, le lecteur trouvera en partie annexe les conventions de transcription adoptées dans le cadre de notre recherche.

\section{Spécificités phonologiques}

Il s'agit de mettre en exergue les spécificités liées à la prosodie. Cette notion "désigne tous les aspects de la parole non liés à l'identification des segments, en particulier les faits d'accentuation lexicale, d'intonation et de rythme" (Vaissière, 2015: 99). Il convient de souligner que ces aspects suprasegmentaux jouent un rôle capital dans la communication, ils ont une fonction expressive dans les échanges linguistiques.

\subsection{Accentuation}

On rencontre sous la rubrique 'accent' des phénomènes très divers, ce qui est souvent source de confusion. En général, il s'agit de phénomènes d'ordre phonique qui consistent à donner un certain relief à telle ou telle partie de la chaîne parlée (syllabe, mot, syntagme, etc.) en faisant contraster un segment particulier avec le reste de l'énoncé. (Encyclopédie Universalis, 2020)

En d'autres termes, l'accentuation correspond à la mise en relief d'une ou plusieurs syllabes; elle se manifeste par une prononciation plus intense et une durée d'émission relativement longue.

Ces accents d'insistance sont particulièrement présents dans le discours du professeur et de la journaliste:

J. "vous n'avez jamais eu de DOUTES pendant cette période>".

J. "cette semaine+un plan de DECONFINEMENT qui sera extrêmement strict".

P. "c'est un modèle qui est en PARTIE+un modèle en train de mourir".

P. "Hydroxychloroquine et l'azithromycine c'est les SEULS médicaments qui sont utilisés+les SEULS+les SEULS qu'utilisent les médecins". 


\subsection{Allongement}

L'allongement vocalique est marqué par une prononciation des voyelles avec une durée d'émission relativement longue et supérieure à la moyenne. Cet aspect prosodique est notamment présent dans les propos du professeur qui se caractérisent par une certaine spontanéité; ce procédé prosodique permet d'effectuer de courtes pauses de réflexion.

P. "La chloroquine donne des résultats clini:ques et radiologi:ques satisfaisants:et:en deuxième temps rédui:t la durée du partage viral".

P. "on est arrivé en fin de parcours+on a un médicament qui ma:rche".

\subsection{Intonation}

Cet aspect prosodique correspond à une courbe mélodique, une variation de hauteur du ton laryngien portant sur un mot ou une suite de mots. L'intonation correspond à une vibration des cordes vocales qui produisent une sorte de mélodie de la chaîne parlée. Elle se manifeste par des variations de la hauteur de la voix, cette dernière n'est pas constante, elle varie en fonction des types de phrases.

"Les différentes modalités (questions, assertions, injonctions, exclamations) qui reflètent la fonction principale de l'acte de communication sont en français d'abord marquées par l'intonation"' (Shirley, 2001: 21). C'est grâce à l'intonation que l'interlocuteur parvient à mieux percevoir l'intention communicative de l'émetteur (informer, interroger ordonner...), cette montée ou chute de la voix est donc porteuse de sens.

\section{- Intonation montante.}

Les propos de la journaliste se caractérisent essentiellement par une intonation montante. Dans ce cas, l'intonation monte en fin de phrase et traduit un questionnement.

\section{Extraits:}

J. " $<$ C'est peut-être pour ça que vous avez envie d'en parler>”.

J. “<Est-ce que ça veut dire que les pays que vous décrivez se sont embourgeoisés $>$ ”.

\section{- Intonation descendante.}

L'intonation descendante baisse en fin de phrase; elle traduit l'idée de certitude et marque la fin des propos, d'une idée, d'un raisonnement... C'est le cas des phrases déclaratives et impératives. Ce type d'intonation est particulièrement présent dans le discours du professeur qui exprime bien souvent des assertions. Extraits:

$\mathrm{P}$. "et bein écoutez on va commencer à traiter les gens avec la chloroquine+parce que c'est disponible+c'est pas cher et que euh finalement on peut traiter les gens avec ça<". P. "[...] donc les gens sont entrainés comme à l'armée+si vous voulez+donc les gens sont entrainés $<"$. 


\section{Spécificités lexicales}

Dans ce qui suit, nous relevons quelques particularités propres au discours médical médiatisé sur le plan lexical. A travers l'analyse du corpus, il nous a été donné de constater que la terminologie usitée s'inscrit dans une frontière: entre langue générale et langue scientifique.

\subsection{Tics de langage}

Les tics de langage correspondent à des automatismes ou encore à des éléments surreprésentés dans le discours d'un locuteur. Ces particules usitées, de manière inconsciente, permettent en quelque sorte de ponctuer le discours, combler les silences et maintenir la communication.

\section{- La particule "voilà".}

Au milieu du tour de parole comme outil remplaçant la ponctuation usitée à l'écrit. Extraits

P. "les chinois ont PUBLIE assez TOT d'ailleurs [...] écoutez+voilà y a DEUX médicaments testés".

p. "[...] y avait toute une partie du monde qu'on connaissait pas sur laquelle on a fait des déductions et qui étaient pas vraies+voilà: c'est la nature [...]"

\section{- La particule " enfin”.}

Au milieu du tour de parole et sert de mot de liaison sans contenu sémantique précis. Extraits:

P. "[...] la chloroquine marche+ça marche in vitro+enfin:+c'est simple [...]"

P. "[...] il y a la question de l'Europe de l'Ouest+enfin:+la Russie aussi [...]".

\section{- L'expression "si vous voulez".}

Cette expression a particulièrement retenu notre attention; elle est très récurrente dans le discours du professeur qui l'emploie systématiquement tout au long de l'échange.

Cette locution verbale est usitée pour orner le discours, elle sert d'une part à marquer, en quelque sorte, une pause de réflexion et d'autre part impliquer l'interlocuteur dans les propos. Extraits:

P. “ ce que NOUS on a fait depuis le départ+si vous voulez:+les choses étaient assez simples+euh initialement $[\ldots] "$

P. “donc+si vous voulez+on a DEUX choses qui comptent dans ces maladies".

\section{- La particule "euh".}

Usitée en situation d'hésitation où le locuteur tente de trouver le mot juste et/ou la formulation correcte. Ce tic de langage est particulièrement répandu dans le discours du professeur qui se caractérise par une relative improvisation. Extraits: 
P. " euh euh+je pense on sait beaucoup plus de cho:ses/on comprend beaucoup plus de cho:ses".

P. “ euh+donc+d'ailleurs c'est le moment+moi j'ai dit+écoutez+à mes étudiants+aux collaborateurs".

\subsection{Vocabulaire scientifique}

Le vocabulaire scientifique correspond à des termes précis etmonosémiques utilisés dans le domaine de la Science.

Le corpus soumis à l'analyse fait apparaître des termes relavant du champ des sciences médicales en général et des maladies infectieuses de manière spécifique. En effet, force est de remarquer que le discours du professeur regorge de termes scientifiques contrairement à la journaliste qui en fait un usage plutôt limité.

Vocabulaire scientifique du professeur:

p. "hydroxychloroquine, azithromycine, diabète, virus, polyarthrite, rhumatoïde, lupus, myocardites, SARS, antibiotique, anticorps, aspirine, lymphocytes, SIDA, hépatite chronique, PCR, arythmie cardiaque, rougeole, variole, fibrose pulmonaire, scanner, grippe espagnole, plaquenil, incubation, réanimation, immunité, maladies immunologiques et inflammatoires, molécule, viral, cancer, essais thérapeutiques, microbes, bactéries, placébo, contagiosité, écosystème, séroprévalence, infection respiratoire, staphylocoque, pneumocoque, techniques sérologiques".

Vocabulaire scientifique de la journaliste:

J. "coronavirus, anticorps, vaccin, syndrome, troubles intestinaux, tocilizumab, anticorps monoclonal, douleur abdominale, troubles gastro-intestinaux, inflammation cardiaque, myocardite, pneumonie, Covid".

Il convient de signaler que la journaliste s'appuie sur des études réalisées par des équipes de recherche et cite systématiquement la source (autorités de santé en Grande Bretagne, assistance publique des hôpitaux de Paris l'APHP, études américaines).

\subsection{Langage familier}

Dans toute situation de communication, il est important d'adapter son langage à son interlocuteur. De façon générale, le professeur intervient dans un registre plus ou moins standard tout au long de l'entretien. Cependant, il est frappant de constater qu'il fait parfois appel au langage familier correspondant à un registre plutôt relâché. Et du coup, il lève les contraintes et marque une certaine spontanéité et proximité avec son interlocutrice.

À vrai dire, le contexte et l'espace où se déroule l'entretien (lieu de travail du professeur) ainsi que la nature de la diffusion (en différée) ont une influence directe sur la communication. Ces conditions confèrent à l'échange un caractère plutôt naturel et spontané. Extraits: 
P. "quand on vous dit qu'un truc est un poison mortel".

P. "les infections grippales dont tout le monde s'est foutu+on sait pas pourquoi [...] tout

le monde s'en est fichu des infections grippales".

P. "je ne sais pas si vos gosses sont vaccinés+en tout cas je vous le recommande moi”.

\section{Spécificités stylistiques}

La rhétorique est la mise en relief conjointe du sens et de la sonorité au moyen de procédés spécifiques tels que les figures d'analogie, de substitution, d'opposition, d'amplification, d'atténuation et de construction. C'est l'art de convaincre, d'impressionner et de bien parler; autrement dit, elle correspond à l'utilisation originale de la langue où l'on joue avec les mots et les constructions de phrases pour frapper les esprits.

Force est de remarquer que nombre de figures de style s'incrustent dans le discours du professeur, notamment la métaphore et l'hyperbole. Ce qui confère à son discours un caractère plus expressif.

\subsection{Métaphore}

La métaphore est souvent rapprochée de l'analogie et de la comparaison. Toute interprétation d'une métaphore passe par un raisonnement analogique, où nous utilisons ou inventons des traits qui unissent la métaphore à sa source (Von Malmborg, 2005: 101).

Le professeur n'hésite pas à embellir et orner son discours scientifique au moyen de la métaphorisation, ce procédé stylistique correspond à une comparaison directe où les termes sont pris au sens figuré. Extraits:

P. "je ne sais d'où est sortie encore cette espèce de fantaisie".

P. "non: on peut tout imaginer+on peut avoir de l'imagination sur tout + mais c'est de la science fiction".

P. "la BATAILLE contre le coronavirus".

P. "la grippe espagnole c'est le grand guignol qui fait peur aux gens".

\subsection{Hyperbole}

Pour marquer ses émotions, le professeur recourt à l'hyperbole, une figure d'amplification, qui correspond à la mise en relief d'une idée au moyen d'une expression exagérée. Extraits:

P. 'l'histoire de l'hydroxychloroquine c'est l'histoire la plus fantasque que je n'ai entendu en médecine+de ma vie".

P. "la société est à bord de la crise de nerfs".

P. "ne pas faire de vaccin à la sauvage".

P. "je ne rentre pas dans une espèce de Folie terrifiante".

P. "y a un de mes amis qui était venu pour nous aider: qui a fait une BOUFFEE délirante". 


\subsection{Expressions idiomatiques}

Les expressions idiomatiques sont des constructions linguistiques dont la signification ne résulte pas de l'analyse compositionnelle de leurs constituants. En d'autres termes, la phrase est porteuse de sens et non pas les mots pris isolément. Ces locutions imagées sont propres à une langue et culture donnée, d'où la nécessité de se méfier de leur traduction.

Pour être mieux compris, la journaliste et le professeur n'ont pas manqué de manier des expressions idiomatiques courantes et familières en contextes appropriés. Ces constructions figées et poétiques donnent plus de couleur et de force à leur propos et provoquent un effet de métaphorisation. Extraits:

J. "vous avez l'impression de leur avoir vendu du rêve" (signifiant promettre quelque chose qu'on ne réalisera pas).

P. "ne pas chercher midi à quatorze heures" (signifiant aller à l'essentiel et éviter la complication).

P. "ça c'est une autre paire de manches" (signifiant c'est une autre affaire).

\section{Stratégies discursives de la journaliste}

\subsection{L'interruption}

Dans les faits, il arrive fréquemment que B s'empare de la parole alors que A n'a apparemment aucune intention de la lui léguer: on dit alors que B interrompt A (Constantin de Chanay \& Kerbrat-Orecchioni, 2010: 56).

L'interruption est considérée comme une tentative d'intervention de la part de la journaliste, qui peut être volontaire ou par inadvertance, au cours du tour de parole du spécialiste. Bien souvent, l'intention de la journaliste est de poser une question, demander une explication ou un éclaircissement, obligeant ainsi son interlocuteur à céder le tour de parole.

\section{Extrait 1}

P. “on est arrivé à une espèce de délire polémique qui est complètement surréaliste y a quelques semaines on n'est pas sorti de ça les uns les autres en prennent un peu raison+on peut donc en parler".

J. “/mais c'est peut-être parce que vous voulez en parler>”.

\section{Extrait 2}

P. "alors y a des choses qui ont besoin de changer+qu'il y ait quelqu'un qui les incarne+moi j'ai pas fait exprès quand j'incarne j'incarne".

J. “/Vous n'avez pas fait exprès+ça vous déplaît pas>”.

\subsection{L'Anticipation}

Il s'agit d'interruptions coopératives où l'initiative semble venir de la part de l'interlocuteur. Cette stratégie est indispensable à la progression de l'échange communicatif 
comme le souligne Kerbrat-Orecchioni, "la coopération et le conflit sont deux composantes également nécessaires à la poursuite du dialogue" (Kerbrat-Orecchioni, 1992: 148).

Il arrive que la journaliste s'implique davantage dans l'entretien et coopère au bon déroulement des échanges. Elle interrompt le discours du professeur, renforce ses propos, anticipe le contenu qui suit, ce qui oblige son interlocuteur à abandonner son tour avant la fin. La stratégie de renforcement mise en place est du type "faire écho" où "l'interlocuteur reprend un segment de la phrase du locuteur en place afin de manifester son approbation" (Béal, 1993: 87). Cette contribution représente une marque d'intérêt et abonde dans le sens du locuteur, comme l'illustre le premier extrait:

\section{Extrait 1}

P. "y a aussi la question de l'Europe de l'Ouest".

J. “/l'Europe de l'Ouest et les États-Unis”.

Par ailleurs, dans le second type de renforcement " même si le locuteur en place est capable de finir sa phrase tout seul, il arrive que l'interlocuteur prenne les devants, ce qui est une manière de montrer qu'il a lu la pensée de l'autre" (Béal, 1993: 88).

\section{Extrait 2}

P. "on a ce qu'on appelle une aversion au risque+on supporte plus le risque donc".

J. “/on s'est affolé”.

\subsection{Le chevauchement}

"Dans le cadre d'une analyse des interactions verbales, les moments où un locuteur est 'physiquement' à la fois en situation d'émission et de réception se limitent aux moments de chevauchement de parole" (Bigot, 2002: 24). Le chevauchement est une stratégie délibérée adoptée par la journaliste qui intervient au cours du tour de parole du professeur, ce qui fait l'effet d'une sorte de superposition de propos des deux interlocuteurs.

\section{Extrait 1}

J. "ça veut dire que c'est une histoire financière quand même+pas forcément scientifique $\mathrm{xxx}$ ".

P. "xxx financière et culturelle".

\section{Extrait 2}

P. "TOUS les médecins du monde+dans $57 \%$ des cas+utilisent azithromycine hydroxychloroquine que vous le recommandiez ou pas/".

J. "mais comment vous l'expliquez qu'en France xxx".

P. "xxx en France mais attendez". 


\subsection{Requête réitérée}

Pour vérifier l'exactitude de l'information, la journaliste attend une deuxième confirmation de la part du chercheur. Nous ressentons ainsi une forte insistance de la journaliste afin de récolter des informations bien précises, garantir une intercompréhension mutuelle et éviter tout malentendu linguistique. Extrait:

J. "donc grâce à votre traitement vous avez trois fois moins de morts c'est ça+++".

P. "+++oui oui".

J. "votre traitement a permis qu'il y ait trois fois moins de morts".

P. "oui oui".

J. "des gens qui SANS votre traitement seraient morts".

P. "oui".

\subsection{L'enchaînement}

L'enchaînement conversationnel correspond à la mise en exergue de la structuration séquentielle. Lors de son intervention, la journaliste tente d'assurer au mieux la gestion de l'alternance des tours de paroles ainsi que l'organisation locale et globale des échanges.

Nous distinguons deux types d'enchaînement, à savoir:

- Enchaînement par opposition.

Marque une stratégie de conflit qui fait progresser le dialogue, d'où l'usage de la conjonction de coordination "mais" en début du tour de parole.

J. "mais alors+comment vous expliquez".

J. "mais vous n'avez pas eu de DOUTES durant cette longue période>".

J. "mais vous n'entendez pas les critiques>".

\section{- Enchaînement par addition.}

Il se manifeste à travers l'usage de la conjonction de coordination "et" en début du tour de parole et sert à mieux relancer la communication.

J. "et qu'est-ce que vous avez pensé de ces théories>".

J. "et la pandémie pour vous+elle est finie>".

J. "et là-dessus vous avez une crainte<".

\subsection{Vulgarisation scientifique}

Nous réservons le terme vulgarisation au traitement de l'information comme transposition d'un langage technique, le plus souvent hermétique, en langage accessible au plus grand nombre grâce à des procédés tels que la paraphrase, l'analogie ou l'inférence (Zappalà, 1997: 181).

La journaliste joue un rôle important dans la vulgarisation et la médiatisation alternant entre langage scientifique et langage usuel explicatif. Elle tente, autant que faire se peut, de 
simplifier le discours du scientifique à travers des formules qui introduisent des reformulations et paraphrases (c'est-à-dire, autrement dit, vous voulez dire, on dit aussi...).

Dans bien des cas, la journaliste oblige son interlocuteur à faire un détour avant de poursuivre une réflexion, s'assure qu'elle a bien compris et demande des informations complémentaires.

Extrait 1

P. "c'est une maladie infectieuse aiguë: quand vous êtes guéris vous immuns".

J. "ça veut dire QUE quand on a eu le coronavirus on l'a plus".

\section{Extrait 2}

P. "les interventions humaines peuvent changer la hauteur du pic + si vous voulez+la dynamique de l'épidémie, c'est une dynamique de l'épidémie qui est juste la dynamique des épidémies+c'est comme ça".

J. "ça veut dire que du point de vue sanitaire+scientifique+confinement ou pas confinement ça n'aurait pas changé tant que ça".

\subsection{Procédés régulateurs}

"Les régulateurs contribuent à étayer, à soutenir le discours du locuteur sans attendre qu'il en manifeste le besoin, en allant au-devant de ses désirs d'approbation" (De Gaulmyn, 1987: 209).

Dans notre corpus, nous avons relevé nombre de régulateurs, ces derniers constituent des indices d'une participation active, des signaux d'écoute, d'implication, des marqueurs d'engagement émis par la journaliste permettant de gérer au mieux la discussion. Nous y avons relevé quelques marqueurs, à savoir: "hmm" et "oui".

Extrait

P. "regardez+vous voyez+au moment où je dis ça".

J. "hmm".

P. "regardez la Chine c'est fini+c'est la fin de la pandémie".

J. "hmm".

P. "vous savez+je crois qu'il faut pas confondre".

J. “/oui".

\section{Stratégies discursives du spécialiste}

\subsection{Expression de l'accord}

"L'accord est une condition locale au développement i.e. la progression de l'interaction" (Moeschler, 1985: 170). Cet acte réactif témoigne à la fois de la dimension coopérative et dynamique de l'échange.

L'interaction s'appuie sur le principe de collaboration et s'oriente vers la recherche 
de la convergence. L'expression de l'accord est un moteur puissant des échanges entre interlocuteurs; ces derniers affichent une adhésion et des objets partagés en ayant recours à des marqueurs linguistiques, tels que " oui”, “parfaitement”, "tout-à-fait”, “c'est sûr”, etc.

Extrait 1

J. "en fait+on manque de créativité+quoi".

P. "oui+et puis $[\ldots]$ on a une vision qui est une vision tunnellaire".

\section{Extrait 2}

J. "dans ce BATIMENT où vous êtes en contact permanent avec des malades+parce que vous les soignez+parce que vous les traitez+le PERSONNEL soignant+POURTANT+n'a pas contracté la maladie"

P. “oui+c'est sûr+c'est sûr".

\subsection{Expression du désaccord}

A la base de l'argumentation il y a toujours deux opinions, deux parties qui s'affrontent qui cherchent à se convaincre mutuellement. Le désaccord y est ainsi présenté comme l'essence même de la communication, le point de départ de tout échange (Bellemmouche et al., 2016)

En d'autres termes, le désaccord est une condition d'émergence et de développement de l'activité argumentative, c'est l'expression d'une divergence de pensées, de positions, de points de vue entre les partenaires de l'échange.

\section{Extrait 1}

J. "vous dites aux médecins n'écoutez pas FORCEMENT les décisions qui sont pri:ses"

p. "non non $[\ldots]$ je peux pas dire ça $[\ldots]$ j'ai pas le droit de dire ça".

\section{Extrait 2}

J. “vous n'avez pas le sentiment de l'avoir minimisé+d'avoir PENSE que ça serait moins important que ça ne l'est devenu>".

P. "vous savez+je vous suggère de vous méfier+c'est seulement quand on aura les comptes qu'on saura".

\subsection{Absence de prédiction}

Malgré la demande insistante de la part de la journaliste, il est frappant de remarquer que le professeur refuse catégoriquement de prédire, il l'a d'ailleurs réitéré à plusieurs reprises dans différents tours de parole. Extrait:

J. "votre intuition>".

P. "je fais pas part de mes intuitions+mes intuitions sont pour moi".

J. "Et dans combien de temps ça sera derrière nous>".

P. "je ne fais pas de pari+vous me demandez de faire des prédictions + je vous dis dix fois je fais pas de prédiction". 


\subsection{Affirmation réitérée}

Afin de mieux appuyer ses propos, mettre l'accent sur les informations jugées pertinentes et produire un effet de persuasion sur son interlocutrice, le professeur recourt à la réitération de mots et expressions clés.

Extrait 1

P. "NEUF CENT MILLE personnes traitées par hydroxychloroquine".

J. "aux mêmes doses>".

P. "aux mêmes doses+c'est des doses que j'utilise depuis des années+NEUF CENT MILLE personnes".

Extrait 2

P. "C'est banal comme forme $+c$ 'est une forme banale $+i$ arrive que cette forme ne soit pas respectée mais c'est banal”.

\subsection{Auto-questionnement et réflexivité}

L'auto-questionnement représente une stratégie discursive incontournable couramment employée dans le cadre scientifique où le chercheur est invité à se questionner lui-même. C'est par le biais de cette démarche mentale qu'il parvient à stimuler sa réflexion, mieux organiser sa pensée et appréhender un ensemble complexe de savoirs, comme en témoignent ces extraits.

P. "si le coronavirus disparaît maintenant comme beaucoup de maladies saisonnières $>+s$ 'il disparaît maintenant $>+s$ 'il revient l'année prochaine $>+$ on verra bien s'il revient l'année prochaine >".

P. 'C'est une maladie nouvelle qu'on connaît pas+est-ce qu'il y a un aspect qu'on connaît pas après cet épisode immunitaire $>[\ldots]$ qu'est ce qu'il y a après ça $>$ ”.

\subsection{Expression de la certitude}

L'expression de la certitude est une caractéristique intrinsèque à tout échange verbal. Au niveau communicatif, le chercheur adopte une attitude d'assurance en soi garantissant la fiabilité, la précision, la véracité et la justesse des informations transmises, comme l'illustrent ces extraits:

P. “j'ai le droit de m'exprimer scientifiquement+parce qu'on est protégés depuis le moyen â:ge++les universitaires ont une liberté de parole qui est protégée [...]+qui est inscrite dans la CONSTITUTION".

P. "on sait+nous+traiter les maladies contagieuses+on sait le faire".

\subsection{Expression de l’incertitude}

Parallèlement, le chercheur manifeste une position de doute, de réticence et se montre plutôt sceptique et perplexe au sujet d'un nombre de propositions et contenus informa- 
tionnels. L'expression de l'incertitude est marquée par des hésitations, des allongements vocaliques, des pauses plus ou moins longues et des verbes d'opinion du type “ je crois, je pense..."

\section{Extrait 1}

J. "est ce que c'est GRACE au confinement que ça a moins tué>”.

P. “j'en sais rien: la causalité+la causalité+la cause des modifications $+\mathrm{j}$ 'en sais rien du tout".

\section{Extrait 2}

J. "il n'y a pas une question de géographie ou de température>".

P. "je+je crois pas + je sais pas".

\subsection{Distanciation critique}

Le développement de la relativisation et de l'esprit critique se manifeste par " la mise en évidence de nouveaux raisonnements scientifiques mettant en cause des pratiques médicales enracinées où la résurgence d>anciennes pratiques abandonnées par la science moderne en sont des exemples" (Zappalà, 1997: 05).

A vrai dire, le savoir médical n'est pas toujours absolu, dans bien des cas, il présente des limites, il est donc à relativiser. C'est pourquoi, le professeur ne manque pas d'adopter une attitude de distanciation critique visant à susciter davantage le débat et la réflexion.

\section{Extrait}

P. “ il n’y a pas un médecin qui n'ait pas de doutes+il n'est pas un chercheur qui n'a pas de dou:tes + il n'y a pas un chercheur qui a que des certitudes + c'est pas raisonnable bien sûr+tout le monde a des dou:tes+je suis sceptique d'ailleurs on me l'a beaucoup reproché + je suis sceptique par nature parce que je suis un scientifique $+y$ a pas de scientifique qui ne soit pas sceptique+quand on cesse d'être sceptique on a basculé à la religion+on arrête d'être un scientifique+un scientifique est toujours prêt à remettre les choses en doute/".

\subsection{Fonction phatique}

Il y a des messages qui servent essentiellement à établir, prolonger ou interrompre la communication, à vérifier si le circuit fonctionne [...], à attirer l'attention de l'interlocuteur ou à s'assurer qu'elle ne se relâche pas (Jakobson, 1963: 217).

Nombre de particules et expressions marquant la fonction phatique sont largement présentes dans le discours du professeur. La finalité de cette stratégie discursive consiste à assurer le maintien de la communication ainsi que la vérification de la recevabilité de la réponse fournie par le professeur. 
- La particule "d'accord".

P. "on a comparé notre étude à toutes les études publiées+d'accord++alors on fait des appariements entre les cas".

P. "avant de changer QUELQUE CHOSE il faut bien réfléchir+parce que les bénéfices

à espérer sont très faibles+toute la question du risque est une question de bénéfice d'accord $>$ donc si on est".

- L'expression "vous voyez".

P. "d'ailleurs vous voyez bien+qu'à la fin+vous ferez le compte+y aura moins de cas".

P. "l'hydroxychloroquine a un rôle au début et à la fin+vous voyez".

- Les formules du type "écoutez, regardez”.

P. "écoutez+je sais pas+c'est un peu trop joué sur les mots".

P. "regardez l'Histoire vous verrez".

\subsection{Stratégies argumentatives}

L'argumentation considère l'interlocuteur, non comme un objet à manipuler mais comme un alter ego auquel il s'agira de faire partager sa vision. Agir sur lui, c'est chercher à modifier les diverses représentations qu'on lui prête, en mettant en évidence certains aspects des choses, en en occultant d'autres, en en proposant de nouvelles (Grize, 1990: 41).

Tout au long de son intervention, le professeur cherche à persuader et à faire partager son point de vue avec méthode et rigueur intellectuelle. Pour ce faire, il recourt à diverses stratégies argumentatives, à savoir:

- Argumentation numérique.

Pour valider ses propos, le spécialiste communique des données scientifiques chiffrées et statistiques. Extrait:

P. "la chloroquine+l'hydroxychloroquine c'est les médicaments les plus PRESCRITS au MONDE après l'aspirine+c'est vendu en 2019+36 MILLIONS de comprimés".

P. "on fait 100000 tests +1 'on a des vraies données+[...] on était à 4000 tests par jour".

\section{- Argumentation logique.}

Le chercheur fait appel tout simplement au raisonnement logique de son interlocutrice. Extrait:

P. "le nombre de cas touchés peut être différent+en fonction des mesures qu'on a prises + le nombre de gens qu'on a traités + si vous baissez la période pendant laquelle on PORTE le virus:+vous diminuez le nombre de cas".

P. "le problème des crises c'est qu'il faut accepter de prendre des risques+parce qu'il y a quelque chose de nouveau et qu'on sait pas gérer".

- Explication argumentative.

Ce procédé argumentatif consiste à expliquer une notion, un principe, un phénomène... en ayant l'intention de mieux l'éclairer. Extrait: 
P. “/je vais vous donner une idée de la contagiosité+les maladies les plus CONTAGIEUSES que l'on est connues c'est+pour l'instant la rougeole et la variole+[...] on estime que ce qu'on appelle le R0 qui est une fantaisie mathématique qui permet de donner un chiffre pour comprendre+c'est quelque chose qui se trouve entre 12 et 20 c'est-à-dire pour chaque cas que vous avez en période d'épidémie vous avez entre 12 et 20 personnes qui vont être contaminées".

- Argumentation causale. S'appuie sur les causes d'un phénomène, sur les principes que les faits ont une cause et produisent des effets (on parle de la mise en relation cause à effet). Extraits:

P. "la baisse de cellules lymphocytes dans le sang+quand vous avez moins de lymphocytes+le risque de mortalité est COLOSSAL".

P. "les gens qui ont une polyarthrite rhumatoïde ont beaucoup moins de Covid parce qu'ils prennent du plaquenil".

\section{- Argumentation par modalisation.}

Ce procédé rend compte de la position du locuteur par rapport à ses propos, au moyen de phrases injonctives et de termes exprimant le devoir et la nécessité.

Le professeur recourt spécifiquement au modalisateur " il faut":

P. " il faut faire attention à faire des vaccins comme ça à la sauva:ge+il faut pas faire ça+[...] il faut vraiment l'évaluer correctement".

P. "y a des myocardites + il faut les surveiller+il faut être attentif".

\section{- Argumentation par généralisation.}

Pour faire admettre une thèse ou renforcer une proposition, le chercheur recourt à la généralisation.

P. "En tout cas les épidémies ont communément une forme de cloche [...] une accélération+un pic et une diminution".

P. "TOUS les médecins ont déjà prescrit du plaquenil dans leur vie+TOUS les médecins connaissent des gens qui ont des maladies inflammatoires".

\section{- Argumentation par comparaison.}

Pour faciliter la compréhension, l'argumentateur s'appuie sur la mise en relation entre deux phénomènes, faits ou réalités, situations etc.

P. "si le coronavirus disparaît maintenant comme beaucoup de maladies saisonnières

[...] regardez le SRAS ça c'est arrêté en juillet 2003 puis il n'y a plus jamais eu".

\section{- Argument d'autorité.}

Pour donner plus de force à son argumentation, le spécialiste fait référence aux déclarations d'une autorité, d'experts et de spécialistes.

P. "les autorités chinoises c'est-à-dire tout le groupe y compris les représentants du gouvernement+y compris les médecins qui avaient mené la lutte contre le SRAS ont fait une déclaration $[\ldots]$ la chloroquine donne des résultats clini:ques+radiologi:ques satisfaisants". 


\subsection{Procédés vulgarisateurs}

Cette stratégie discursive déployée par le professeur indique qu'il est “ conscient de l'écart qui existe entre le langage scientifique et la compréhension d'un public tout venant" (Charaudeau, 2008: 21). Cette pratique est indispensable pour retenir l'attention du public, l'inciter à suivre le fil de la discussion, assurer la transparence du discours et garantir la transmission des contenus cognitifs émis par le scientifique.

- Recours à la définition.

P. “[...] c'est ce qu'on appelle la séroprévalence+la présence d'anticorps chez les gens".

P. "l'hydroxychloroquine est un modulateur de l'immunité [...]+c'est le TRAITEMENT des maladies immunologiques et inflammatoires".

P. "c'est en période d'INCUBATION entre le moment où vous êtes exposé et le moment où vous êtes malade que vous soyez symptomatique ou pas".

P. "les myocardites qui sont des inflammations du cœur sont: des complications qu'on observe dans les maladies virales en général".

- Recours à l'illustration.

P. “toutes les données initiales que l'on a sont des idées que j'appelle tunnellaire+c'està-dire vous ne testez qu'une seule hypothèse+et parce que vous avez testé qu'une seule hypothèse vous ne pensez pas qu'il y ait d'autres hypothèses valides + je vous donne un exemple les antibiotiques sont pour l'essentiel des molécules qui sont faites par les microbes pour se battre entre eux+et donc on a testé ces molécules contre les bactéries+mais pendant des années on les a jamais testées sur les virus donc on savait pas que ça marchait contre les virus".

\section{- Recours à l'explication.}

P. "on est dans un changement du mo:nde très important+on a un changement de modèle c'est-à-dire que+je vous explique $+c$ 'est-à-dire la course en avant technologique en disant les vieux médicaments sont moins bons que les nouveaux médicaments qui est dans la pensée de beaucoup".

P. "la courbe en cloche est une courbe habituelle $+c$ 'est comme ça que se passent beaucoup d'épidémies+elles mon:tent pour des raisons qu'on comprend pas elles deviennent de plus en plus contagieuses+de plus en plus sévères+et puis elles diminuent".

\section{Conclusion}

Au terme de notre recherche, nous espérons avoir contribué, ne serait-ce que très modestement, à éclairer la question relative aux stratégies conversationnelles qui sous-tendent le discours médical médiatisé. À vrai dire, l'analyse de discours est incontestablement un domaine riche et inépuisable; et plus exactement, l'analyse du discours médical représente un champ d'investigation inédit qui a suscité davantage notre curiosité intellectuelle.

A travers la recherche entreprise, nous avons tenté de mettre en exergue les spécificités discursives propres à ce type de communication et avons analysé de près la dynamique interactive et les comportements langagiers des interlocuteurs. De surcroît, cette recherche 
a mis en évidence l'importance des stratégies argumentatives déployées dans ce cadre communicatif spécifique.

L'analyse conduit à conclure que les stratégies, mises en œuvre de manière consciente, intentionnelle et coopérative, donnent du sens aux échanges verbaux et leur confèrent un caractère à la fois formel et spontané. Il importe de souligner également que cette organisation discursive se caractérise par une certaine complémentarité des rôles des participants, assurant des positionnements distincts, ce qui contribue à assurer une progression au niveau local et global.

Bien entendu, ce travail de recherche a permis d'aboutir à un certain nombre de conclusions, cependant notre projet est susceptible de s'orienter vers d'autres pistes de réflexion tout à fait pertinentes. En effet, dans les travaux futurs, nous envisageons d'appréhender le discours médical médiatisé dans toute sa complexité, élargir le corpus et nous focaliser notamment sur sa visée pragmatique.

Dans cette perspective, nous considérons que les médias ne se limitent pas à décrire des faits scientifiques; ils dépassent la fonction informationnelle et assurent parallèlement une fonction pragmatique dans la mesure où ils tentent d'agir sur autrui et sur le monde.

\section{Références bibliographiques}

BÉAL, Christine. 1993. "Les stratégies conversationnelles en Français et en anglais" in Langue Française, $\mathrm{n}^{\circ}$ 98,79-106: <https://www.persee.fr/doc/lfr_0023-8368_1993_num_98 ___5835> [15/09/2020].

Bellemmouche, Hacene, Elodie Baklouti \& Abdelrhamn Alokla. 2016. "Manifestations discursives du désaccord en domaine français" in Cahiers de praxématique, $\mathrm{n}^{\circ} 67:<\mathrm{https}: / /$ doi.org/10.4000/praxematique.4346> [26/09/2020].

Bennaissa, Azzedine, 2018. "Les Pratiques d'interaction dans le discours médical Médiatisé" in Pensée Méditerranéenne, vol.7, n², 286-298: <https://www.asjp.cerist.dz/en/article/62004> [22/09/2020].

Bigot, Violaine. 2002. Lieux et modes de construction de la relation interpersonnelle en contexte didactique: analyse d'interactions verbales en cours de Français langue étrangère. Paris, Presses de Paris3-Sorbonne Nouvelle.

Charaudeau, Patrick. 2008. "De la situation et du contrat de communication" in La médiatisation de la science. Bruxelles, De Boeck, 11-22.

Constantin de Chanay, Hugues, \& Catherine Kerbrat-Orecchioni. 2010. "Les interruptions dans le débat médiatique: une stratégie actionnelle" in Pratiques, linguistique, littérature, didactique, Interactions et corpus oraux, $\mathrm{n}^{\circ} 147-148,83-104:$; journals.openedition.org/ pratiques/1614> [05/10/2020].

De Gaulmyn, Marie-Madeleine. 1987. Les régulateurs verbaux: le contrôle des récepteurs. Lyon, Presses Universitaires.

ENCYCLOPÉDIE UNIVERSALIS. 2020. <www.universalis.fr > encyclopedie > accent-linguistique $>[26 / 11 / 2020]$. 
FANNY, Rinck. 2010. "L'analyse linguistique des enjeux de connaissance dans le discours scientifique. Un état des lieux" in Revue d'anthropologie des connaissances, $\mathrm{n}^{\circ} 3$, vol. 4, 427-450: <https://www.cairn.info/revue-anthropologie-des-connaissances-2010-3-page-427. htm $>[26 / 11 / 2020]$.

Grize, Jean-Blaize. 1990. Logique et langage. Paris, Ophrys.

JAKOBSON, Roman. 1963. Essais de linguistique générale. Paris, Seuil.

Kerbrat-Orecchioni, Catherine. 1992. Les interactions verbales II, Paris, Armand Colin.

Kerbrat-Orecchioni, Catherine. 2016. Les actes de langage dans le discours; théorie et fonctionnement. Paris, Armand Colin.

Moeschler, Jacques. 1985. Argumentation et conversation. Éléments pour une analyse pragmatique du discours. Paris, Crédif / Hatier.

SHIRLEY, Vinter. 2001. "La prosodie, structure d'accueil des compétences langagières", in Revue française de linguistique appliquée, vol 6, 21-34: <https://www.cairn.info/revue-francaise-de-linguistique-appliquee-2001-1-page-21.htm $>$ [10/12/2020].

Siouffi, Gilles \& Raemdonck VAN. 2007. 100 fiches pour comprendre la linguistique. Paris, Bréal.

VAISSIÈRe, Jacqueline. 2015. "Prosodie" in La phonétique: <https: //www.cairn.info/la-phonetique--9782130653356.htm> [30/11/2020].

VION, Robert. 1992. La communication verbale, analyse des interactions. Paris, Hachette.

Von Malmborg, Gunila. 2005. "Régularités et répartition. Analyse de quelques métaphores dans L'Express et Le Monde" in Mots, les langages des politiques, $\mathrm{n}^{\circ} 79,101-115<\mathrm{https}$ :// doi.org/10.4000/mots.1664> [02/07/2021].

Zappalà, Annick. 1997. "La Médecine Médiatisée: entre la médicalisation du social et la socialisation de la science" in HERMES, n²1, 181-190. Suisse, Université de Fribourg<http:// documents.irevues.inist.fr/bitstream/handle/2042/15053/HERMES_1997_21_181. pdf; sequence $=1>[25 / 12 / 2020]$.

\section{Annexe}

\section{Conventions de transcription}

\begin{tabular}{ll}
\hline J. abréviation du mot "journaliste" & / Interruption \\
\hline P. abréviation du mot "professeur" & xxx Chevauchement \\
\hline$>$ Intonation montante & + Pause courte \\
\hline$<$ Intonation descendante & ++ Pause moyenne \\
\hline :Allongement vocalique & +++ Pause longue \\
\hline Majuscule: Accentuation & "euh" Hésitation $[\ldots]$ Troncation \\
\hline
\end{tabular}


